\title{
A Review of the Results of the Very Long Chain Fatty Acid Analyses for X-linked Adrenoleukodystrophy at the Biochemical Genetics Laboratory of the Institute of Human Genetics-National Institutes of Health, Manila
}

\author{
Esphie Grace F. Fojas, Judy S. Manliguis, Cristine P. Lopez, Sheryl V. Decena, \\ Kathleen Gayl D. Fonacier and Mary Anne D. Chiong \\ Biochemical Genetics Laboratory, Institute of Human Genetics, National Institutes of Health, University of the Philippines Manila
}

\begin{abstract}
Background. X-linked adrenoleukodystrophy (X-ALD) is a progressive genetic disorder affecting the metabolism of very long chain fatty acids in the adrenal glands, spinal cord and white matter of the nervous system. It is an inherited metabolic storage disease whereby a defect in a specific enzyme results in the accumulation of very long-chain fatty acids (VLCFA) that are harmful to some cells and organs. VLCFA analysis for confirmation of X-linked adrenoleukodystrophy is one of the most requested tests among the send-out laboratory services of the Biochemical Genetics Laboratory at the Institute of Human Genetics. This paper aims to review the clinical characteristics and the results of the VLCFA analysis of the patients whose samples we received for testing.
\end{abstract}

Methods. Overseas tests samples received by the Biochemical Genetics Laboratory for VLCFA from 2002-2016 were included. The details of the patients were collated in an overseas tests database and was the main source of the data for this study. The results of the VLCFA tests sent to the Kennedy Krieger Institute and The Children's Hospital at Westmead were inputted into the said database. Descriptive statistics was utilized in order to examine the clinical and biochemical data of the patients.

Results. The results showed that out of the 54 samples submitted to our laboratory, 19 (35\%) of the samples received from male patients suspected to have X-ALD yielded positive results and another 10 (19\%) females were found to be carriers. Visual defect followed by deteriorating mental status were the most frequent indications for VLCFA testing.

Conclusion. Having a significant diagnostic yield of 54\%, early diagnosis of $\mathrm{X}$-linked adrenoleukodystrophy in our population is important so that proper management that could prevent the progression of the disease could be timely instituted.

Corresponding author: Esphie Grace F. Fojas, DRDM

Biochemical Genetics Laboratory

Institute of Human Genetics

National Institutes of Health

University of the Philippines Manila

623 Pedro Gil St., Ermita, Manila 1000 Philippines

Telephone: +6325261725 local 114

Email: esphiefojas@gmail.com
Key Words: inborn errors of metabolism, peroxisomal disorders, very long chain fatty acid analysis, $X$-linked adrenoleukodystrophy

\section{Introduction}

The Biochemical Genetics unit at the Institute of Human Genetics (IHG), National Institutes of Health, University of the Philippines Manila is the only laboratory in the country which caters to the diagnosis and research of inborn errors of metabolism (IEM). IEM is a group of diseases that arise from a block in a metabolic pathway, and represent a special challenge in general and pediatric practice as they frequently present with acute, life-threatening crisis that require immediate specific intervention. ${ }^{1}$ The laboratory offers locally-available and overseas tests for the analysis of samples from patients who are considered to have IEM. The most common IEM groups are amino acid disorders, organic acid disorders, fatty acid oxidation disorders, mitochondrial disorders, lysosomal storage disorders, and peroxisomal disorders.

Peroxisomes are organelles where many oxygendependent reactions take place to protect the cell against oxygen radicals. Dysfunctions in peroxisomal metabolism include defects in pristanic acid, intermediates of bile acid synthesis, $\alpha$-oxidation of 3-methyl fatty acids (e.g. phytanic acid), biosynthesis of ether lipids (e.g. plasmalogens), glyoxylate detoxification, and biogenesis and $\beta$-oxidation of very long chain fatty acids (VLCFA). ${ }^{2}$

Fatty acids are essential components of the body. They are classified according to carbon $(\mathrm{C})$ chain length and number of double bonds. Very long chain fatty acids are fatty acids with chains longer than C20 (C>20). The number of double bonds correspond to the degree of saturation (i.e. saturated, monounsaturated/one double bond, or polyunsaturated/two or more double bonds). ${ }^{3}$ X-linked adrenoleukodystrophy (X-ALD) is the most common peroxisomal disorder affecting the metabolism of very long chain fatty acids. ${ }^{4,5}$ It is caused by mutations in the $A B C D 1$ gene which is located at the X-chromosome and belongs to a superfamily of human ATP-binding cassettes for import of VLCFAs into peroxisomes.5,6 To date, 750 mutations have been identified, while the overall estimated prevalence of X- 
Table 1. Summary of Results

\begin{tabular}{|c|c|c|c|c|}
\hline \multirow{2}{*}{ Count } & \multirow{2}{*}{$\begin{array}{c}\text { Samples Sent to Children's Hospital } \\
\text { at Westmead (plasma) }\end{array}$} & \multicolumn{2}{|c|}{ Samples Sent to Kennedy Krieger Institute } & \multirow{2}{*}{ Total } \\
\hline & & Plasma & Dried Blood Spots & \\
\hline Number of Males & 19 & 5 & 11 & 35 \\
\hline Number of Females & 8 & 9 & 2 & 19 \\
\hline Total Number of Samples Sent & 27 & 14 & 13 & 54 \\
\hline Number of Positive Males & $8(30 \%)$ & $5(100 \%)$ & $6(46 \%)$ & $19(35 \%)$ \\
\hline Number of Carriers & $0(0 \%)$ & $9(100 \%)$ & $1(8 \%)$ & $10(19 \%)$ \\
\hline Number of Normal Results & $19(70 \%)$ & $0(0 \%)$ & $6(46 \%)$ & $25(46 \%)$ \\
\hline
\end{tabular}

ALD in newborns is $1: 17,000 .^{5}$ The phenotype of the progressive childhood form was first described by Siemerling and Creutzfeld in 1923, and Moser et al. first showed in 1980's that X-ALD was associated with increased VLCFA levels in plasma. Since then, this assay has been widely used as a screening tool for X-ALD. Increase in the VLCFA levels is the primary metabolic marker for this disease, and the other predominant peroxisomal metabolites such as plasmalogens, phytanic acid, pristanic acid, and bile acids remain within normal range. ${ }^{2,5,7}$ In the literature, two ratios are also further suggested for the diagnosis of $\mathrm{X}-\mathrm{ALD}$, that is, $C 24: 0 / C 22: 0$ and $C 26: 0 / C 22: 0 .{ }^{5}$ Men having only one X-chromosome develop X-ALD symptoms; women having two X-chromosomes with only one affected are usually referred as carriers and develop milder form of the disease. ${ }^{5}$

VLCFA analysis is an offered overseas test by the Institute of Human Genetics' Biochemical Genetics laboratory and are being sent to Kennedy Krieger Institute in Maryland, USA and The Children's Hospital at Westmead in Sydney, Australia.

This paper aims to review the clinical characteristics and the results of the very long chain fatty acid analyses of patients suspected to have X-ALD which were forwarded to our laboratory for overseas testing. The results of this study shall provide information regarding the statistics of the disease in terms of its frequency and most prevalent clinical profiles which may help improve the diagnosis of X-ALD among the Filipino population.

\section{Methods}

Overseas tests samples received by the Biochemical Genetics Laboratory come with uniform request forms. Details of the patients such as date of birth, specimen type, and checklist of presenting symptoms with provided blank space for those not specified on the checklist, were included in this form. These data were either filled-up by the hospital which forwarded the sample, the patients' parents/ guardians; or extracted by a Biochemical Genetics Laboratory staff from an attached/forwarded medical abstract. The clinical information in this study is limited to those indicated in these forms. These data were then collated in an overseas tests database of the Biochemical Genetics laboratory and was the main source of the data for this study. Patients whose samples were sent for VLCFA from year 2002-2016 were included. Diagnostic yield refers to the percentage of the positive samples over the total number of samples.
From 2002-2005, 14 plasma samples were sent to the Kennedy Krieger Institute (KKI) which used a validated laboratory method for VLCFA testing using this sample type. Results were presented in $\mu \mathrm{g} / \mathrm{mL}$ showing patient's/carrier's result, normal controls (range from their pool of normal samples), X-ALD heterozygote (range of their results for carriers) and X-ALD hemizygote (range of their results for positive males). No samples were sent from 20062010 to this laboratory. From 2011, a validated laboratory method for VLCFA testing using dried blood spot samples became the preferred type of sample for send out to KKI because of easier handling during transport. From this year up to 2016, a total of 13 dried blood samples were sent and tested. Results were presented in pmole/spot showing patient's/carrier's result, normal range (range from their pool of normal samples), X-ALD range (range of results for their X-ALD positive patients) and X-ALDH (range of results for their carrier). The total samples sent to Kennedy Krieger Institute for both plasma and dried blood samples was 27.

A total of 27 plasma samples were sent to The Children's Hospital at Westmead from 2005-2015. The laboratory made use of a validated laboratory protocol for VCLFA testing for plasma samples. Results were presented in $\mu \mathrm{mol} / \mathrm{L}$ showing the patient's results and the reference ranges for very long chain fatty acids.

The results of the VLCFA tests from Kennedy Krieger Institute and The Children's Hospital at Westmead were inputted into the overseas tests' database. Descriptive statistics was utilized in order to examine the clinical and biochemical data of the patients. A total of 5 samples were listed in the database without any detail except last names, hence were excluded in this study.

\section{Results}

From 2002-2016, a total of 54 samples for VLCFA analysis were sent out cumulatively to The Children's Hospital at Westmead and Kennedy Krieger Institute. Out of this sum, 35 were males (age range: 1-month old-15 years old) and 19 were females (age range: 2 years old-61 years old). The number of samples sent out for each institution are shown in Table 1.

A sum of 19 male patients out of the total 54 samples (35\%) turned out positive with high levels of VLCFA beyond the reference range for the corresponding ages. An additional $10(19 \%)$ samples were found to have levels of VLCFA that were compatible with being carriers for the 
disease, making an overall diagnostic yield of $54 \%$. Out of the 10 carriers, $50 \%$ of them were mothers of the confirmed patients with X-ALD. There were 25 patients whose VLCFA levels fell in the normal range (46\%) (Figure 1).

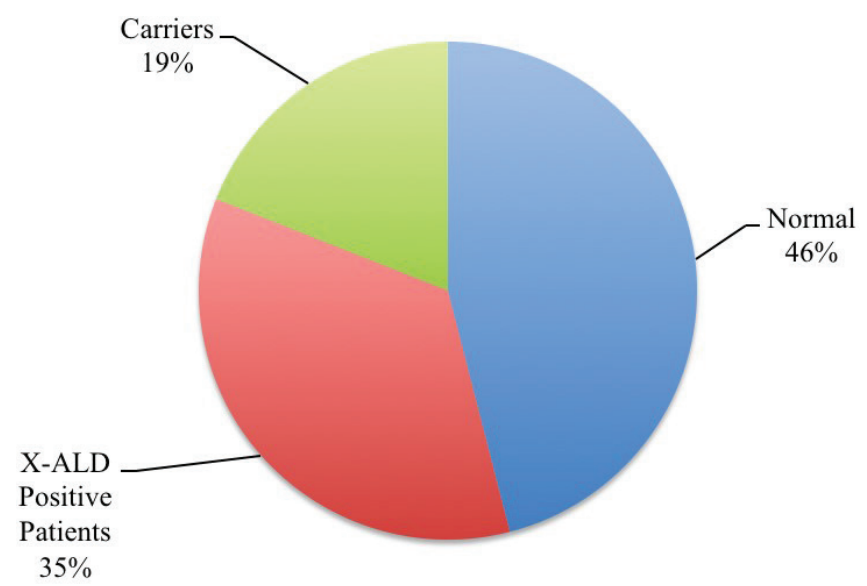

Figure 1. X-ALD sample distribution for all samples, 20022016.

The patients who had normal VLCFA ranges had presenting symptoms which were mostly neurologic as written in the clinical indications submitted. These included global developmental delay, demyelinating disease, degenerative disease, numbness, tingling sensation, congenital muscular dystrophy, Zellweger syndrome, Addison's disease, hyponatremia, and irregular heart rhythm. Others were tested due to a relative confirmed to have X-ALD relative, with or without specified symptoms.

The age range for the males confirmed to have above normal VLCFA levels was 5-15 years old with a mean age of 8 years $( \pm 3 S D)$. Sixteen patients $(84 \%)$ were $4-10$ years of age and three patients (16\%) were $11-15$ years of age. For the carriers, the age range was 28-61 years old, with an average of 40 years of age $( \pm 10$ SD).

The clinical indications written on the request forms and the distribution frequency of the symptoms in all the males confirmed to have X-ALD are shown in Table 2. Among the carriers, only one specified the reason for testing which was having a child confirmed to have X-ALD. Only 5 patients out of 19 positive males had cranial MRI results written on their request forms which all indicated a white matter disease.

The results of hexacosanoate (C26:0), the chief marker of X-ALD, and the C24/C22 and C26/C22 ratios for the plasma samples sent to Children's Hospital at Westmead are shown in Table 3. A total of eight positive males were tested in this institution. No carriers were detected by this laboratory. For the positive males, $\mathrm{C} 26: 0$ had a mean of $3.73 \mu \mathrm{mol} / \mathrm{L}$ (normal value: $0.33-1.13 \mu \mathrm{mol} / \mathrm{L})$. The mean of $\mathrm{C} 24 / \mathrm{C} 22$ and $\mathrm{C} 26 / \mathrm{C} 22$ ratios among these patients were 1.91 (normal value: 0.601.02 ) and 0.10 (normal value: 0.007-0.023), respectively.
Table 2. Clinical indications for patients with confirmed XALD after VLCFA testing

\begin{tabular}{lc}
\hline \multicolumn{1}{c}{ Symptoms } & $\begin{array}{c}\text { Frequency of Presentation } \\
\text { in X-ALD Positive Patients }\end{array}$ \\
\hline Visual defect/blurring vision & $8(20 \%)$ \\
Deteriorating mental skills & $6(15 \%)$ \\
White matter disease (on MRI result) & $5(12 \%)$ \\
Behavioral change (hyperactivity) & $4(10 \%)$ \\
None/not specified & $4(10 \%)$ \\
Ataxia & $3(8 \%)$ \\
Deafness & $3(8 \%)$ \\
Speech delay & $3(7 \%)$ \\
Seizures & $2(5 \%)$ \\
Vomiting & $1(3 \%)$ \\
No clinical symptoms specified & $1(2 \%)$ \\
\hline
\end{tabular}

Table 3. Results of C26:0, C24/C22, and C26/C22 for positive plasma samples

\begin{tabular}{lcc}
\hline $\begin{array}{c}\text { Samples Sent to The Children's } \\
\text { Hospital at Westmead (plasma) }\end{array}$ & $\begin{array}{c}\text { X-ALD Positive } \\
\text { Patients } \mathrm{N}=8\end{array}$ & $\begin{array}{c}\text { Reference Range } \\
(\mu \mathrm{mol} / \mathrm{L})\end{array}$ \\
\hline C26:0 $(\mu \mathrm{mol} / \mathrm{L})$ Range & $1.75-4.44$ & $0.33-1.13$ \\
C26:0 $(\mu \mathrm{mol} / \mathrm{L})$ Mean & 3.73 & \\
Standard Deviation & 0.93 & $0.60-1.02$ \\
\hline C24/C22 Range & $1.6-2.34$ & \\
C24/C2 Mean & 1.91 & $0.007-0.023$ \\
Standard Deviation & 0.22 & \\
\hline C26/C22 Range & $0.05-0.14$ & \\
C26/C2 Mean & 0.10 & \\
Standard Deviation & 0.03 & \\
\hline
\end{tabular}

Table 4. Results of C26:0, C24/C22, and C26/C22 from plasma samples of positive males

\begin{tabular}{lccc}
\hline $\begin{array}{c}\text { Samples Sent to Kennedy } \\
\text { Krieger Institute (plasma) }\end{array}$ & $\begin{array}{c}\text { X-ALD Positive } \\
\text { Patients' Results } \\
\mathbf{N}=5\end{array}$ & $\begin{array}{c}\text { Normal } \\
\text { Controls }\end{array}$ & $\begin{array}{c}\text { X-ALD } \\
\text { Hemizygote }\end{array}$ \\
\hline C26:0 $(\mu \mathrm{g} / \mathrm{mL})$ Range & $1.22-2.03$ & $0.24 \pm 0.14$ & $1.30 \pm 0.45$ \\
C26:0 $(\mu \mathrm{g} / \mathrm{mL})$ ) Mean & 1.64 & & \\
Standard Deviation & 0.38 & & $1.71_{ \pm} 0.23$ \\
\hline C24/C22 Range & $0.68-2.37$ & $0.78 \pm 0.10$ & \\
C24/C22 Mean & 1.92 & & \\
Standard Deviation & 0.63 & & \\
\hline C26/C22 Range & $0.12-0.20$ & $0.01 \pm 0.003$ & $0.07 \pm 0.03$ \\
C26/C22 Mean & 0.15 & & \\
Standard Deviation & 0.03 & & \\
\hline
\end{tabular}

For the plasma samples sent to Kennedy Krieger Institute, five males and nine carriers tested positive, totaling fourteen patients. For the positive males, the mean C26:0 was 1.64 (normal value: $0.24 \pm 0.14$ ), the mean $\mathrm{C} 24 / \mathrm{C} 22$ was 1.92 (normal value: $0.78 \pm 0.10$ ), and the mean $\mathrm{C} 26 / \mathrm{C} 22$ ratio was 0.15 (normal value: $0.01 \pm 0.003$ ). These results including the reported value ranges for X-ALD hemizygotes are presented in Table 4.

One positive male had a normal C24/C22 ratio of 0.68 , the lower limit for the range. However, his C26:0 result and $\mathrm{C} 26 / \mathrm{C} 22$ ratio were above the reference ranges which confirmed the diagnosis.

For the carriers, mean C26:0 was 0.74 (normal value $0.24 \pm 0.14)$, mean $\mathrm{C} 24 / \mathrm{C} 22$ was $1.53(0.78 \pm 0.10)$, and mean $\mathrm{C} 26 / \mathrm{C} 22$ was $0.03(0.01 \pm 0.003)$. The results including the 
reported value ranges for X-ALD heterozygotes are presented in Table 5.

One of the carriers also had a normal C26:0 value of 0.32 , the second lowest value in the cohort, but her C24/C22 and C26/C22 ratios were increased which led to the probability that she could be a carrier. Another carrier had normal values for C26:0 (0.22) and C26/C22 ratio (0.02), both the lower limits for their ranges. However, her C24/C22 was increased and by pedigree analysis, she is an obligate heterozygote for X-ALD. These examples highlight the possibility of normal VLCFA results for carriers and the importance of $\mathrm{C} 24 / \mathrm{C} 22$ and $\mathrm{C} 26 / \mathrm{C} 22$ ratios and pedigree analysis in the analysis of carrier samples.

For the dried blood spot samples sent to Kennedy Krieger Institute, six males and one carrier tested positive for X-ALD. Only the C26:0 results were reported for this group; C24/C22 and C26/C22 ratios were not specified. The mean C26:0 for males confirmed to have X-ALD was 1.64 (normal value: 0.06-0.51). These results including the reported value ranges for X-ALD hemizygotes are presented in Table 6.

The value for the carrier was 0.60 (normal value: 0.060.51 ). The result including the reported value ranges for $X-$ ALD heterozygotes is presented in Table 7.

Table 5. Results of C26:0, C24/C22, and C26/C22 from plasma samples of carriers

\begin{tabular}{lccc}
\hline $\begin{array}{l}\text { Samples Sent to Kennedy } \\
\text { Krieger Institute (plasma) }\end{array}$ & $\begin{array}{c}\text { Carriers' Results } \\
\mathbf{N}=\mathbf{9}\end{array}$ & $\begin{array}{c}\text { Normal } \\
\text { Controls }\end{array}$ & $\begin{array}{c}\text { X-ALD } \\
\text { Heterozygote }\end{array}$ \\
\hline C26:0 $(\mu \mathrm{g} / \mathrm{mL})$ Range & $0.22-1.09$ & $0.24 \pm 0.14$ & $0.68 \pm 0.29$ \\
C26:0 $(\mu \mathrm{g} / \mathrm{mL})$ ) Mean & 0.74 & & \\
Standard Deviation & 0.32 & & $1.30 \pm 0.19$ \\
\hline C24/C22 Range & $1.25-1.79$ & $0.78 \pm 0.10$ & \\
C24/C22 Mean & 1.53 & & \\
Standard Deviation & 0.2 & & \\
\hline C26/C22 Range & $0.02-0.12$ & $0.01 \pm 0.003 \pm 0.02$ & \\
C26/C22 Mean & 0.03 & & \\
Standard Deviation & 0.03 & & \\
\hline
\end{tabular}

Table 6. Results of C26:0 from the dried blood spots samples of positive males

\begin{tabular}{lccc}
\hline $\begin{array}{c}\text { Samples Sent to Kennedy Krieger } \\
\text { Institute (dried blood spot) }\end{array}$ & $\begin{array}{c}\text { X-ALD Positive } \\
\text { Patients' Results } \\
\text { N=6 }\end{array}$ & $\begin{array}{c}\text { Normal } \\
\text { Range }\end{array}$ & $\begin{array}{c}\text { X-ALD } \\
\text { Range }\end{array}$ \\
\hline C26:0 (pmole/spot) Range & $0.65-2.91$ & $0.06-0.51$ & $0.86-5.2$ \\
C26:0 (pmole/spot)) Mean & 1.64 & & \\
Standard Deviation & 0.8 & & \\
\hline
\end{tabular}

Table 7. Result of C26:0 from the dried blood spot samples of a carrier

\begin{tabular}{lccc}
\hline $\begin{array}{c}\text { Samples Sent to Kennedy Krieger } \\
\text { Institute (dried blood spot) }\end{array}$ & $\begin{array}{c}\text { Carrier's } \\
\text { Result N=1 }\end{array}$ & $\begin{array}{c}\text { Normal } \\
\text { Range }\end{array}$ & $\begin{array}{c}\text { X-ALDH } \\
\text { Range }\end{array}$ \\
\hline C26:0 (pmole/spot) Range & 0.6 & $0.06-0.51$ & $0.36-3.1$ \\
C26:0 (pmole/spot)) Mean & 0.6 & & \\
Standard Deviation & $\sim$ & & \\
\hline
\end{tabular}

This review was able to include 54 samples over a course of 15 years. This was a very low sample volume considering the high birth rate in the Philippines of around 1.7 million births in $2014^{8}$, and given the fact that the worldwide prevalence of X-ALD is 1:17,000. But despite the low sample volume, there was a high diagnostic yield in this series which points to a great possibility that our local patients may be undiagnosed or underdiagnosed for this condition. On the other hand, the results also indicate that physicians could recognize well enough the cluster of symptoms for this disease given the high frequency of a positive elevation of VLCFAs among patients and/or carriers suspected to have the disease.

$\mathrm{X}$-ALD is transmitted through X-chromosomal inheritance, hence it primarily affects males. The name "adrenoleukodystrophy" was introduced by Dr. Michael Blaw: 'adreno' refers to the adrenal glands; 'leuko' refers to the white matter of the brain, and 'dystrophy' means abnormal growth or development. It has three main types in affected males: childhood cerebral form, adrenomyeloneuropathy (AMN), and Addison disease. ${ }^{5}$ The two most common forms affecting males are AMN (60\%) and the adult cerebral form $(35 \%) .{ }^{9}$

The childhood cerebral form which occurs in boys aged 4-10, but never before 3 years old, usually present with school failure, behavioral changes, visual/hearing impairment, intellectual regression, ataxia, adrenal insufficiency, leukodystrophy, and seizures. Some studies report intermittent vomiting. ${ }^{10,11}$ Neuroimaging of $85 \%$ of the affected males show magnetic resonance imaging (MRI) that show the characteristic pattern of symmetrical enhanced T-2 signal in the parieto-occipital region with contrast enhancement at the advancing margin. ${ }^{5}$ AMN usually manifests in the late twenties with progressive symptoms of paraparesis, sphincter disturbances, sexual dysfunction, and often, impaired adrenocortical function; while Addison disease presents with adrenocortical insufficiency between two years of age and adulthood without evidence of neurologic disability, however may develop later some degree of neurologic disability. Affected females or carriers develop neurologic manifestations that resemble AMN but have later onset and have milder disease. ${ }^{12}$ While they are normally tested at 40-50 years of age, a recent study showed that more than $80 \%$ of women develop symptoms after 60 years of age. It has likewise been noted that AMN in women with X-ALD is often misdiagnosed as multiple sclerosis. When VLCFA concentrations are normal, women who are suspected to have X-ALD should be tested using molecular techniques because $15 \%$ of carriers have normal plasma VLCFA. ${ }^{5,13}$

Based on the age range of the patients in this study, majority of the patients had the childhood cerebral form 
while a few presented with the adolescent form. No adult cerebral X-ALD case was documented. The age range for carrier detection in this series seems to be in keeping with what has been reported in the literature where the mean age for the diagnosis of carriers was reported to be at around 40 years of age. ${ }^{5}$ All of the patients with abnormal VLCFA presented with the commonly recognized symptoms of $\mathrm{X}$ ALD. Visual defect or blurring vision was the most common followed by the deterioration in mental skills and white matter disease on cranial imaging. On the other hand, hyperactivity which was also seen in the patients, could be one of the documented reasons for delay in diagnosis ${ }^{5}$. Lesser frequencies were observed for deafness, speech delay ataxia, seizures, and vomiting. For the carriers, no presenting symptoms were specified, except for one mother who was tested because she had a child who had a positive VLCFA testing. The data on the patients' adrenal functions were not specified.

The concentration of C26:0 for all our patients and/or their corresponding $\mathrm{C} 24 / \mathrm{C} 22$ and $\mathrm{C} 26 / \mathrm{C} 22$ ratios were likewise pathognomic for X-ALD, having higher amounts compared to the ranges for normal controls. Genetic testing and genetic counseling were recommended to the affected families.

\section{Conclusion}

This paper highlights that X-ALD could be a prevalent inborn error of metabolism in the country given a diagnostic yield of $54 \%$ among the patients suspected to have the disease and among carriers. Awareness of this frequency may help physicians ascertain the patients earlier as this can prevent severe neurologic and physical complications of the disease. Treatment options such as bone marrow transplantation may halt the progression of the disease if diagnosed early.

This study has been limited by the clinical features that were given by the requesting physicians and the biochemical staff had no access to their clinical records. It is therefore recommended to study further the clinical, radiologic, and endocrine manifestations of the patients and carriers in the tested cohort to fully characterize the expression of the disease in the Filipino population. The Biochemical Genetics laboratory is currently setting up VLCFA testing so that it can be made locally available in the near future.

\section{Statement of Authorship}

All authors have approved the final version submitted.

\section{Author Disclosure}

All authors declared no conflict of interest.

\section{Funding Source}

None.

\section{References}

1. Fumic K, Bilic K. Laboratory diagnostics of emergency conditions associated with inborn errors of metabolism. Signa Vitae. 2014; 9(1):70-2.

2. Zschocke J, Hoffman G. Peroxisomal metabolism. In: Zschocke J, Hoffman G, eds. Vademecum Metabolicum Manual of Metabolic Pediatrics, $3^{\text {rd }}$ ed. Germany: Milupa GmbH; 2001. p. 143.

3. Sassa T, Kihara A. Metabolism of very long chain fatty acids; genes and pathophysiology. Biomol Ther. 2014; 22(2):83-92.

4. Grkovic S, Nikolic R, Djordjevic M., et al. X-Linked adrenoleukodystrophy: profiles of very long chain fatty acids in plasma and fibroblasts in eight Serbian patients. Indian J Clin Biochem. 2007; 22(2):118-22.

5. Adrenoleukodystrophy Database (Netherlands) [Online]. October 15, 2015 [cited 2015 Oct]. Available from www.x-ald.nl.

6. ABCD1 ATP binding cassette subfamily D member 1 [Homo sapiens (human)] (US) [Online]. Novermber 5, 2015 [cited 2015 Nov]. NCBI Resources: Gene. Available from www.ncbi.nlm.nih.gov/books/NBK1315.

7. Kennedy CR, Allen JT, Fensom AH, Steinberg SJ, Wilson R. X-linked adrenoleukodystrophy with non-diagnostic plasma very long chain fatty acids. J Neurol, Neurosurg Psychiatry. 1994; 57(6):759-61.

8. Live Births in the Philippines 2014 (Phils.). [Online]. January 26, 2017 [cited 2017 Jan]. Philippine Statistics Authority. Home: Vital Statistics. Available from https://psa.gov.ph/content/live-births-philippines-2014.

9. Moser A, Jones R, Hubbard W, et al. Newborn Screening for X-linked Adrenoleukodystrophy. Int J Neonatal Screen. 2016; 2(4):15-9.

10. Soardi FC, Esquiaveto -Aun AM, Guerra-Junior G, de Lemos-Marini SHV, de Mello MP. Phenotypic variability in a family with X-linked adrenoleukodystrophy caused by the p. Trp132Ter mutation. Arq Bras Endocrinol Metab. 2010; 54(8):738-43.

11. Vargas CR, Coelho D, Barschak A, et al. X-Linked adrenoleukodystrophy: clinical and laboratory findings in 15 Brazilian patients. Genet Mol Biol. 2000; 23(2):261-4.

12. X-linked adrenoleuokodystrophy. (US) [Online]. January 11, 2017 [cited 2017 Jan]. NCBI Resources: Bookshelf. Available from www.ncbi.nlm. nih.gov/books/NBK1315.

13. Tran C, Patel J, Stacy H, et al. Long-term outcome of patients with xlinked adrenoleukodystrophy: a retrospective cohort study. Eur J Pediatr Neurol. 2017; 21(4):600-9. 